\title{
Leiomyosarcoma of the Foot: A case report
}

\author{
by Sutpal Singh, DPM, FACFAS ${ }^{1}{ }^{⿴}$, Chih-Hui (Jimmy) Tsai, DPM ${ }^{2}$, Albert Kim, DPM ${ }^{3}$, \\ Timothy Dailey, $\mathrm{DPM}^{4}$, Vir Nanda, $\mathrm{MD}^{5}$, Rao Paladugu, $\mathrm{MD}^{6}$
}

The Foot and Ankle Online Journal 3 (11): 1

The authors present a case report of a rare leiomyosarcoma. The tumor presented as an indurated mass on the dorsum of the foot. The mass was excised, and further treated by an oncologist. It is important to be suspicious of any large tumor on the dorsum of the foot because of possible malignancies. Early diagnosis and treatment contribute to a better prognosis.

Key Words: Leiomyosarcoma, tumor of the foot, smooth muscle cell tumor.

Published: November, 2010

This is an Open Access article distributed under the terms of the Creative Commons Attribution License. It permits unrestricted use, distribution, and reproduction in any medium, provided the original work is properly cited. @The Foot and Ankle Online Journal (www.faoj.org)

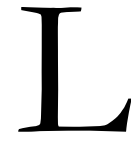
eiomyosarcoma has been recognized for many years. Leiomyosarcomas can be divided into three types based on their site of origin. The most common type is leiomyosarcomas of soft tissues. The best prognosis is cutaneous leiomyosarcoma and arises from the small muscles in the hair follicles. The rarest type is vascular leiomyosarcomas. ${ }^{1}$ It is a type of spindle cell sarcoma, which is a soft tissue tumor, originating from smooth muscle. These tumors most commonly affect the uterus and the abdominal cavity. ${ }^{2}$ It occurs in the fifth to seventh decade and affects more males than females. ${ }^{3}$ Leiomyosarcoma in the foot is relatively rare and infrequently reported. Only 21 cases of leiomyosarcoma in both the foot and ankle have been reported in the literature since $1936 .{ }^{4}$

Address correspondence to: Sutpal Singh, DPM, FACFAS, Chief Ilizarov Surgical Instructor at Doctors Hospital West Covina, Fellow of the American College of Foot and Ankle Surgeons, Private practice in Southern California.

\footnotetext{
${ }^{1}$ Chief Ilizarov Surgical Instructor at Doctors Hospital West Covina, Fellow of the American College of Foot and Ankle Surgeons, Private practice in Southern California.

2 Doctor of Podiatric Medicine (R3), Foot and Ankle Medicine and Surgery, Doctors Hospital of West Covina (PM\&S-36).

${ }^{3}$ Doctor of Podiatric Medicine (R2) Foot and Ankle Medicine and Surgery, Doctors Hospital of West Covina (PM\&S-36).

${ }^{4}$ Doctor of Podiatric Medicine (R1) Foot and Ankle Medicine and Surgery,

Doctors Hospital of West Covina (PM\&S-36).

${ }^{5}$ Oncologist Private Practice in Southern California.

${ }^{6}$ Pathologist at St Mary Regional Medical Center.
}

Approximately 350 spindle cell sarcomas are diagnosed each year and of these only eight percent are leiomyosarcomas. Of these eight percent, only about four percent occur in the foot and ankle. ${ }^{5}$ This approximately equates to 1 case per year. These tumors can present as a non-painful mass which grows larger than normal, can be single nodes or multi nodular. Due to the non-painful nature of this tumor, during its early stages, diagnosis can be greatly delayed. Treatment for subcutaneous leiomyosarcoma is resection and some authors suggest postoperative radiotherapy. ${ }^{1}$

The strongest prognostic factor for survival is the presence of metastasis at diagnosis, with the most common site being the lungs. The prognosis for patients with purely cutaneous leiomyosarcoma is an unusually good $90 \%$ survival. ${ }^{4}$ The patient should then be rescanned every 3 months for the first 2 years and then every 6 months for the next 3 years. ${ }^{4}$ In a case paper by Rifleman, Cronin, and Sage, they reported a recurrence rate of approximately $60 \% .{ }^{6}$ 


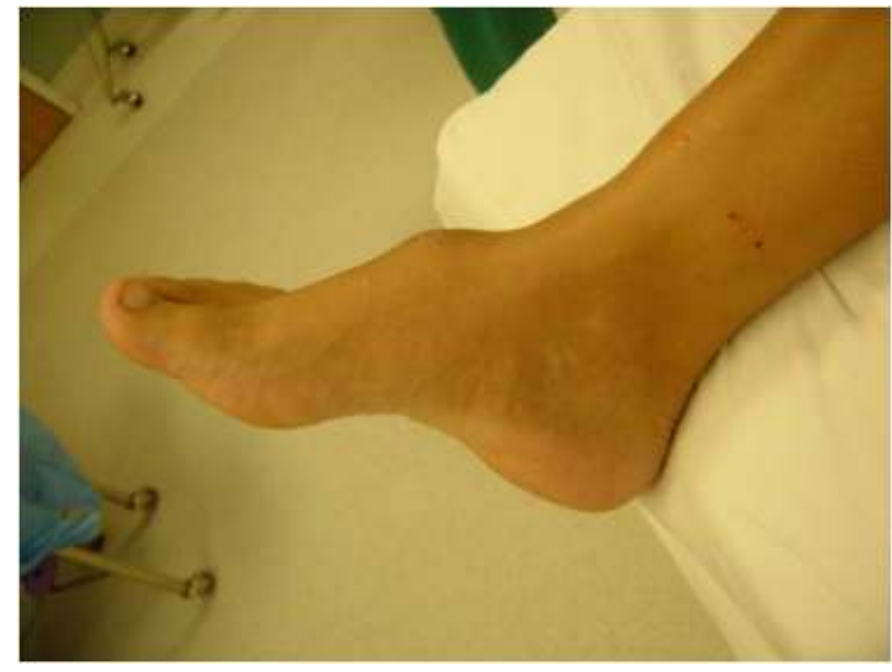

Figure 1 Preoperative view of the foot with a distinctive dorsal mass along the top of the foot.

Here, we report a case of subcutaneous leiomyosarcoma, which was initially diagnosed as a ganglionic cyst and was referred to podiatry by a primary care physician for evaluation. As it turned out, the mass was not a cyst, but a sarcoma.

\section{Case Report}

This is a 33-year-old female who presented to the office with soft tissue swelling for many years. (Fig. 1) It is now a large mass on the dorsum of the right foot and is painful. Her primary care physician had diagnosed it as a ganglion cyst and the patient was referred for an aspiration, which yielded no fluid. The needle aspirate was then sent for tissue analysis. The pathology report indicated that the aspirate cells were not typical of a benign ganglion .(Fig. 2) An open biopsy was scheduled. An magnetic resonance image (MRI) was ordered before biopsy. The MRI showed that there was a well-circumscribed soft tissue mass measuring $3.2 \mathrm{~cm}$ in length and $2.2 \mathrm{~cm}$ in thickness. There was also a fairly uniform signal with increased signal compatible with the soft tissue mass with no destruction of the underlying bones or extension into the deep tissue. (Figs. 3A and 3B)

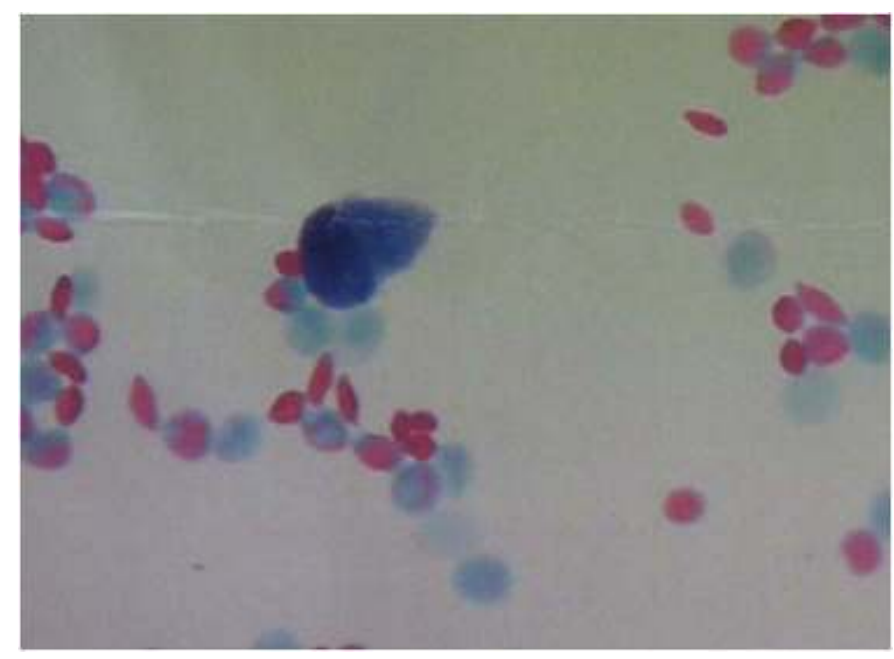

Figure 2 Aspiration biopsy: Labeled soft tissue right foot showing scattered atypical cells.

The computed tomography (CT) scan also confirmed a similar sized mass located subcutaneously along the dorsal aspect of the tarsal bones with uniform CT density, no irregularity, and no extension of the mass lesion or infiltration or destruction of the underlying bones (Fig 4).

\section{Surgical Techniques}

The patient had an open biopsy performed with a small incision overlying the mass down to the subcutaneous level. Several $2 \mathrm{~mm}$ open punch biopsies were taken. The pathology report yielded a high-grade mesenchymal neoplasm consistent with sarcoma. (Fig. 5) The patient had a wide excisional resection of the mass performed at a later date. The patient was placed in a supine position and then a \# 10 blade was used to make an incision over the mass. This was deepened to the subcutaneous tissue. (Figs. 6A and 6B) The mass was noted under and engulfing the extensor hallucis longus tendon. The tissue was then dissected to the deeper levels just superior to the first three rays and corresponding cuneiforms. 

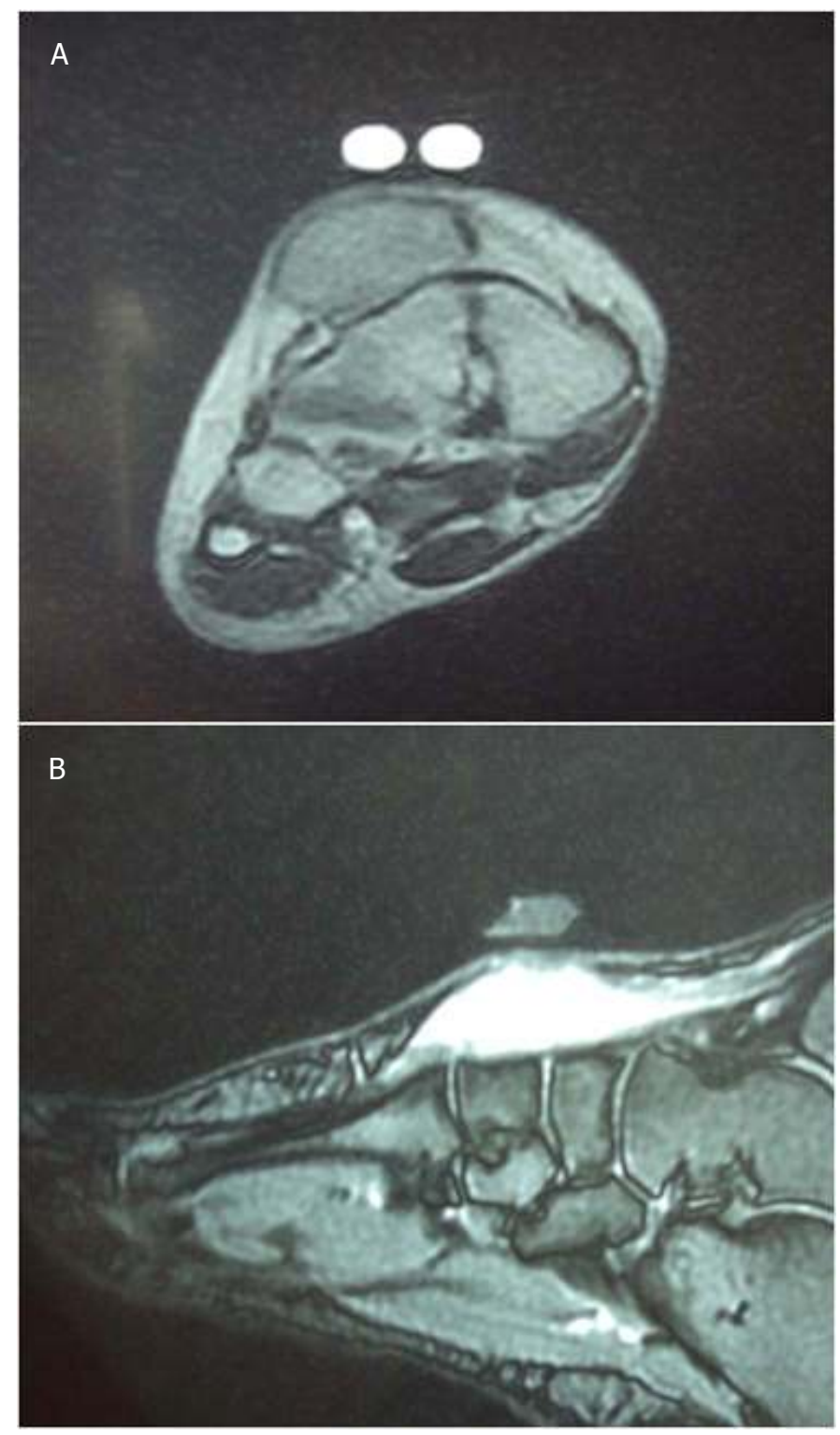

Figure 3A and 3B MRI: Note the soft tissue mass dorsal to the underlying bones. Coronal section $(A)$ and lateral view shows lesion directly over midtarsal joint region. (B)

The entire mass, the involved extensor hallucis longus tendon, and surrounding tissue were all removed and sent for pathological analysis. The area was then copiously irrigated with normal saline and bacitracin and then closed using 3-0 Vicryl and 3-0 Prolene.

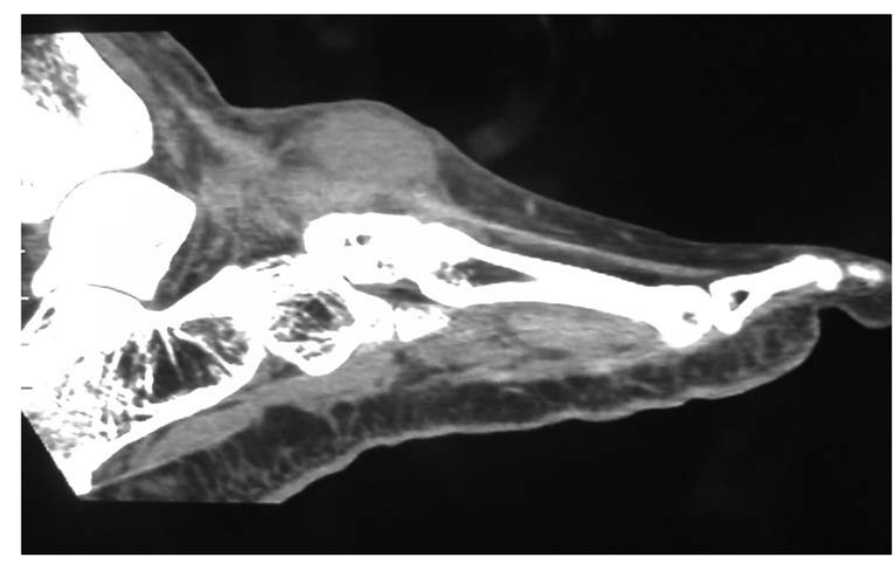

Figure 4 CT scan: Note that the soft tissue mass has not infiltrated the underlying tarsal bones.

\section{Results}

The tumor presented a gray white cut surface and it was unencapsulated. The tumor consisted of diffuse sheets of elongated spindle shape cells with moderate amounts of eosinophilic cytoplasm and elongated nuclei which are hyperchromatic with rounded ends. The neoplastic cells are also arranged in fascicles with the nuclei showing considerable pleomorphism. The mitosis was variable and average 8 per 10 high power fields. Multinucleated tumor giant cells are readily seen. Mitosis is helpful in differentiating these tumors from their benign counterparts. In view of the location of the tumor, secondary changes like pressure necrosis, inflammatory changes due to trauma and focal hemorrhage are also noted. Other areas of the resected tumor show fibrocollagenous adipose, muscle and tendinous tissue with focal areas of infiltration by the malignant tumor cells. Special staining for actin, myoglobin, S-100 protein, vimentin, and CD-57 were also performed. The actin, myoglobin and vimentin were positive, the S-100 was weakly positive and the CD-57 was negative. (Fig. 7) In light of this immunohistochemical and microscopic analysis, a diagnosis of poorly differentiated malignant tumor with features consistent with leiomyosarcoma was made. 


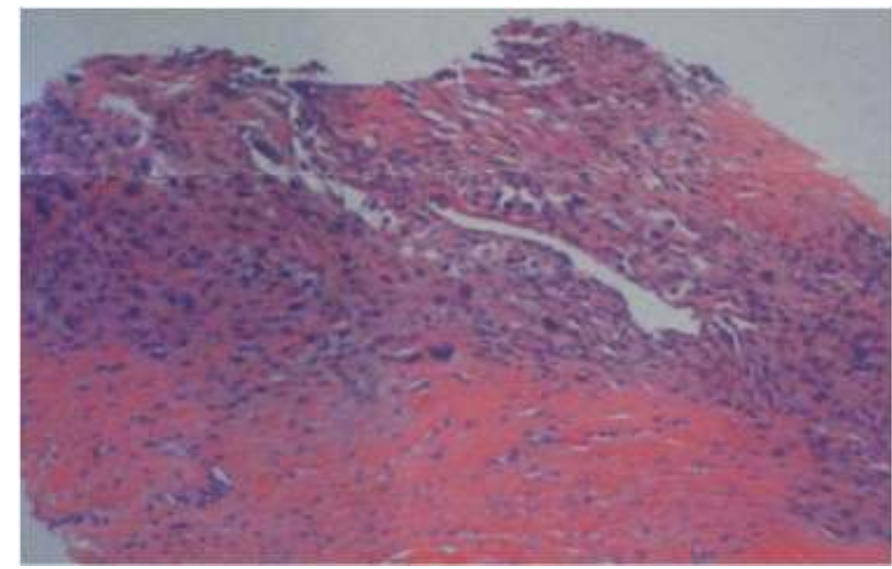

Figure 5 Soft tissue open incisional biopsy: High grade neoplasm consistent with considerable pleomorphism of tumor cells.

\section{Discussion}

Leiomyosarcomas are rare, resistant, malignant soft tissue tumors that originate and mutate from involuntary smooth muscle cells. In this case report, the most likely category of leiomyosarcoma would either be cutaneous or subcutaneous.

Cutaneous leiomyosarcomas are derived from precursor cells associated with the arrector muscles of the hair. Cutaneous leiomyosarcomas often present as a solitary nodule smaller than $2 \mathrm{~cm}$, usually on the extremities, particularly on the hair bearing extensor surfaces and on the lower extremities. The epidermis over the lesion may exhibit a pink, purple, brown, or red discoloration with possible crusting or ulceration. Although a minority of patients are asymptomatic, a majority of patients report pain. ${ }^{1}$

Subcutaneous leiomyosarcomas originate from the muscular walls of veins and arteries. The inferior vena cava and veins of the lower extremities are the most common sites. This type of leiomyosarcoma has a more rapid growth rate than cutaneous leiomyosarcomas.

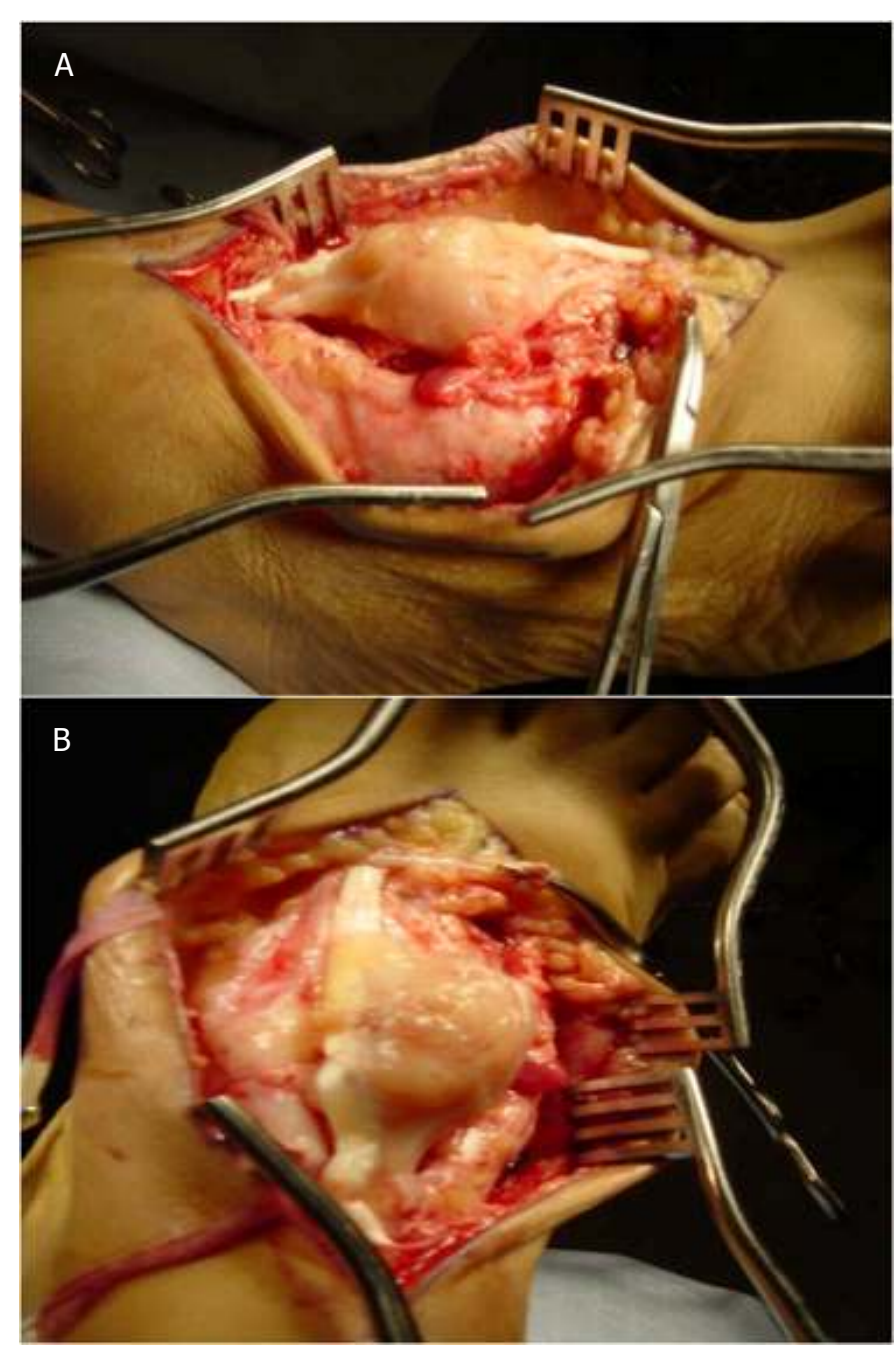

Figure 6A and 6B Soft tissue mass being resected. Note that the extensor hallucis longus is completely infiltrated by the mass. (A) The unencapsulated tumor shows diffuse infiltration of adjacent structures. (B)

Pain and numbness are the most common symptoms in contrast to cutaneous leiomyosarcomas, these lesions may appear well circumscribed. The risk of metastasis is greater than in cases of cutaneous leiomyosarcomas and the risk appears to increase with depth. ${ }^{9,10}$

In this case, the lesion was presumed to be a ganglion cyst from the shape, location, and clinical presentation. The advanced imagining also presented the lesion to be a ganglion cyst because of signal intensities on MRI, as well as the well circumscribed nature. 


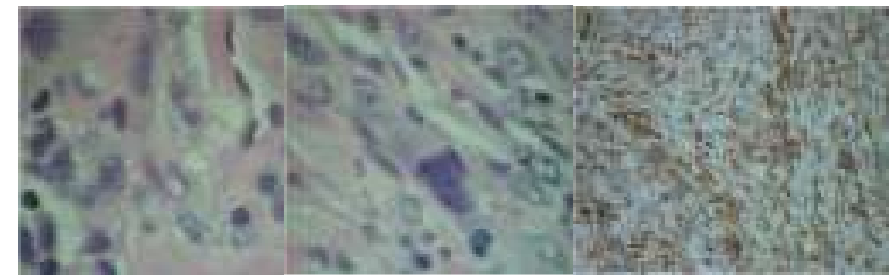

Figure 7 Histopathological findings of the excisional biopsy. Note the tumor giant cell in the middle photograph.

However, this was not the case after aspiration and several biopsies suggesting a different diagnosis. The ultimate diagnosis of leiomyosarcoma was determined after histologic findings and immunohistochemical assays with evidence of neoplastic cells and positive finding for special staining for actin, myoglobin and vimentin, and S-100 protein. ${ }^{11}$

This patient most likely had a subcutaneous leiomyosarcoma due to the well circumscribed nature and deeper location. Although cutaneous leiomyosarcomas are more common on the extensor surfaces of the lower extremities, a cutaneous leiomyosarcoma was ruled out due to the lack of epidermal changes, the larger size of this lesion, and the deeper location. Interestingly, the pathology report noted marked pleomorphism that may categorize this lesion to be pleomorphic leiomyosarcoma, which is a morphologic variant. ${ }^{13}$

The treatment of leiomyosarcomas is directed by its stage, location, size, and patient's age. Since this leiomyosarcoma was well circumscribed and the ability to take a wide excisional resection of the mass was possible, it was determined that the wide excisional resection was the most appropriate treatment. Note that a below the knee amputation is also a viable choice but the patient refused this option. The patient also received radiation therapy and this seems to improve the local control of the disease. ${ }^{12}$ The literature recommends patients should be observed for at least 5 years after the surgery with most recurrence occurring at around the 2 year mark. ${ }^{13,14}$

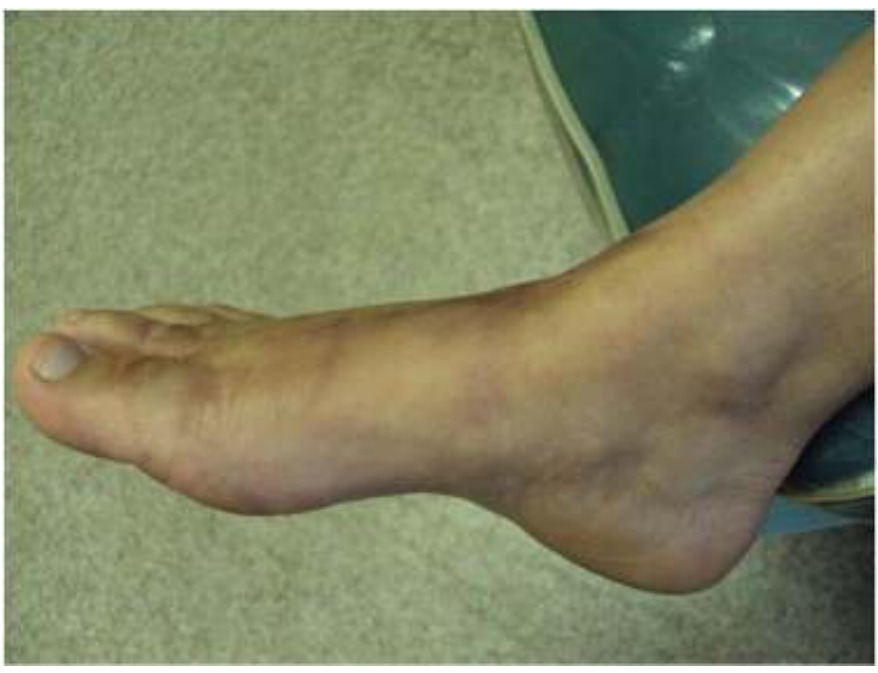

Figure 8 Three year follow up after radiation treatment.

The early detection of this leiomyosarcoma, wide excision, and the radiation therapy has resulted in a satisfactory outcome in this case. (Fig. 8) However, recurrences can occur and it is recommended to have periodic follow ups.

\section{Conclusion}

Though rare in nature, it is important to always be suspicious of a large mass on the dorsal aspect of the foot. Diagnosis of the pathology should be performed in a timely matter. Also, appropriate consultations and radiographic studies should be used in order to examine the extent of involvement.

\section{References}

1. Abed R, Abudu A, Grimer J, Tillman M,Carter R, Jeys L. Leiomyosarcomas of vascular origin in the extremity. Sarcoma 2009.

2. Svarvar C, Böhling T, Berlin Ö, Gustafson P, Follerås G, Bjerkehagen B, Domanski HA, Sundby Hall K, Tukiainen E, Blomqvist C. Clinical course of nonvisceral soft tissue leiomyosarcoma in 225 patients from the Scandinavian sarcoma group. Cancer 2007 109(2).

3. Angeloni M, Muratori F, Magarelli N, Chalidis BE, Ricci R, Rossi B, Maccauro G. Exophytic growth of a neglected giant subcutaneous leiomyosarcoma of the lower extremity. A case report. International Seminars in Surgical Oncology 2008 5:11. 
4. Erin E, Butler M, Anain Jr J. Leiomyosarcoma of the foot. JAPMA 2007 97(6): 475-479.

5. Kransdorf M. Malignant soft tissue tumors in a large referral population: Distribution of diagnoses by age, sex, and location. American J Radiology 1995 164: 129-134.

6. Rifleman GT, Cronin R, Sage R. Leiomyosarcoma of the Cutaneous Tissue. JAPMA 1990 80(4): 222-225.

7. Efstathopoulos N, Lazarettos J, Vassilios N, Efstathios C. Inflammatory leiomyosarcoma of the ankle: A case report and review of the literature. J Foot \& Ankle Surg 2006 45(2):127-130.

8. Bulmer JH. Smooth muscle tumors of the limbs. JBJS 1967 49B(1) 52-58.

9. Fields JP, Helwig EB. Leiomyosarcoma of the skin and subcutaneous tissue. Cancer 1981 47:156-169.

10. Dahl I, Angervall L. Cutaneous and subcutaneous leiomyosarcoma: A clinicopathologic study of 47 patients. Pathologia Europea 1974 9: 307.

11. Suster S. Epithelioid leiomyosarcoma of the skin and subcutaneous tissue: clinicopathologic, immunohistochemical, and ultrastructural study of five cases. Am J Surg Pathol 1994 18: 232-240.

12. Angeloni M, Muratori F, Magarelli N, Chalidis BE, Ricci R, Rossi B, Maccauro G. Exophytic growth of a neglected giant subcutaneous Leiomyosarcoma of the lower extremity. A case report. Int Semin Surg Oncol 2008 May 21(5):11.

13. Tran L, Mark R, Meier R, Calcaterra T, Parker R. Sarcomas of the head and neck. Prognostic factors and treatment strategies. Cancer 1992 70:169-177.

14. Oda Y, Miyajima K, Kawaguchi K, Tamiya S, Oshiro Y, Hachitanda Y, Oya M, Iwamoto Y, Tsuneyoshi M. Pleomorphic leiomyosarcoma: clinicopathologic and immunohistochemical study with special emphasis on its distinction from ordinary leiomyosarcoma and malignant fibrous histiocytoma. Am J Surg Pathol 2001 25(8):1030-1038. 